\title{
Erratum to: CDOM fluorescence as a proxy of DOC concentration in natural waters: a comparison of four contrasting tropical systems
}

\author{
E. Rochelle-Newall • F. D. Hulot • J. L. Janeau • \\ A. Merroune
}

Published online: 13 February 2014

(C) Springer International Publishing Switzerland 2014

\section{Erratum to: Environ Monit Assess \\ DOI 10.1007/s10661-013-3401-2}

The original version of this article unfortunately contained a mistake. There were transcription errors in the row for "Soil leachate (2010)". The range under the column "Absorption $\left(\mathrm{a}_{(355)} \mathrm{m}^{-1}\right)$ " should read 0.1870.8 , and the slope under the column "Absorption versus fluorescence" should read 9.78.

The errors do not change the conclusion of the article. Below is the corrected table.

The online version of the original article can be found at http://dx.doi.org/ 10.1007/s10661-013-3401-2.

E. Rochelle-Newall $\cdot$ J. L. Janeau $\cdot$ A. Merroune IRD-Bioemco, UMR 7618, c/o Soils and Fertilisers Research Institute (SFRI), Dong NgacTu Liem District

Hanoi, Vietnam

E. Rochelle-Newall $(\bowtie)$

IRD-Bioemco, UMR 7618,

UPMC-CNRS-INRA-ENS-IRD-AgroParisTech-Université

Paris-Est Ecole Normale Supérieure, 46 rue d'Ulm,

75005 Paris, France

e-mail: emma.rochelle-newall@ird.fr

F. D. Hulot

Ecologie, Systématique et Evolution, UMR 8079,

Univ. Paris-Sud, Orsay, France 
Table 2 Regressions of absorption versus fluorescence and DOC versus fluorescence for each sample site

\begin{tabular}{|c|c|c|c|c|c|c|c|c|c|}
\hline & $\begin{array}{l}\text { Absorption } \\
\left(a_{(355)} \mathrm{m}^{-1}\right)\end{array}$ & $\begin{array}{l}\text { Fluorescence } \\
\text { (NFIU) }\end{array}$ & $\begin{array}{l}\text { DOC } \\
(\mu \mathrm{M} \mathrm{C})\end{array}$ & \multicolumn{3}{|c|}{$\begin{array}{l}\text { Absorption versus } \\
\text { fluorescence }\end{array}$} & \multicolumn{3}{|c|}{$\begin{array}{l}\text { DOC versus } \\
\text { fluorescence }\end{array}$} \\
\hline & \multicolumn{3}{|c|}{ Range (min-max) } & Intercept & Slope & $r^{2}$ & Intercept & Slope & $r^{2}$ \\
\hline Bach Dang estuary (July 2008) & nd & $5.1-40.2$ & $91.0-144.2$ & nd & nd & nd & -43.2 & 0.51 & 0.75 \\
\hline Bach Dang estuary (April 2009) & $0.27-2.24$ & $2.0-33.2$ & $90.8-158.9$ & -0.36 & 1.76 & 0.93 & -7.6 & 0.18 & 0.16 \\
\hline Bach Dang estuary (November 2011) & nd & $1.7-22.2$ & $86.4-175.5$ & nd & nd & nd & -20.7 & 0.26 & 0.74 \\
\hline $\begin{array}{l}\text { Freshwater lens, New Caledonia } \\
\quad(2007-2008)\end{array}$ & $2.57-60.9$ & $70.7-1308$ & $298-4,277$ & 47.8 & 22.2 & 0.99 & 248.5 & 0.15 & 0.34 \\
\hline Zimbabwe (2010) & $4.28-37.0$ & $43.8-252.2$ & $339.2-2,550.9$ & 35.9 & 5.7 & 0.66 & 28.6 & 0.08 & 0.70 \\
\hline Soil leachate (2010) & $0.18-70.8$ & $0.3-620.4$ & $65.22-5,190.6$ & 49.7 & 9.78 & 0.88 & 12.5 & 0.12 & 0.98 \\
\hline
\end{tabular}

Values in italics are those not significant at the $p=0.05$ level nd not determined 